\title{
Ruptured Left Sinus of Valsalva Aneurysm in a Female Nigerian Septuagenarian
}

\author{
Mohammed Abdullahi Talle ${ }^{1,2 *}$, Faruk Buba ${ }^{1,2}$, Ahmed Abulfathi $^{3}$ and Musa Mohammed Baba ${ }^{1}$ \\ ${ }^{1}$ Department of Medicine, College of Medical Sciences, University of Maiduguri, Nigeria \\ ${ }^{2}$ Department of Medicine, Cardiology Unit, University of Maiduguri Teaching Hospital, Maiduguri, Nigeria \\ ${ }^{3}$ Division of Clinical Pharmacology, Department of Medicine, Faculty of Medicine and Health Sciences, Stellenbosch University, Cape Town, South Africa
}

\begin{abstract}
Sinus of Valsalva Aneurysm (SVA) is a rare cardiac condition that is commonly congenital. Acquired SVA is very rare, resulting from infections affecting the aortic wall, degenerative diseases or traumatic injury to the chest. Left SVAs are usually acquired, especially when associated with rupture into the left heart. We present a case of ruptured left SVA in a female Nigerian Septuagenarian who presented with congestive cardiac failure.
\end{abstract}

Keywords: Sinus of valsalva; Aneurysm; Heart failure; Septuagenarian

\section{Introduction}

Sinus of Valsalva Aneurysm (SVA) is an uncommon cardiac anomaly that may be congenital or acquired. Congenital SVA is more common, and results from weakness of the aortic media at its junction with the annulus fibrosus [1]. The right coronary sinus is the most common site involved as observed in $75 \%$ to $90 \%$ of cases, followed by the non-coronary sinus in $10 \%$ to $25 \%$. Congenital lesion of the left coronary artery is very rare, probably because it does not originate from the bulbar septum [2]. Acquired SVA can result from trauma, endocarditis, syphilis, tuberculosis and connective tissue diseases [3-5].

The reported incidence of SVA ranges from $0.1-3.0 \%$ of all congenital heart defects, accounting for $0.14 \%$ of all open heart surgical procedures, while autopsy series suggests a prevalence of $0.09 \%$ in the general population $[6,7]$.

Most unruptured SVA remain asymptomatic and incidentally discovered during echocardiography. However, the presentation in ruptured SVA is variable, depending among other things the receiving chamber, and whether the rupture is acute or chronic. Ruptured SVA commonly present with continuous murmur, often suspected to be due to patent ductus arteriosus or coronary fistula, or congestive cardiac failure [1]. We present a case of a ruptured SVA in a 70-year old lady presenting with heart failure and mitral incompetence.

\section{Case Presentation}

A 70-year old lady was referred to our unit with a four-week history of progressive shortness of breath, paroxysmal nocturnal dyspnea, orthopnea, cough, abdominal and leg swelling. There was a history of palpitation but no syncope or chest pain. She developed fever a week prior to presentation. She was not known to be hypertensive or diabetic, and had not suffered myocardial infarction, trauma to the chest, stroke or transient ischemic attack.

Physical examination revealed a frail elderly lady comfortably lying in cardiac position with bilateral pitting pedal edema extending to the knees. There were no Oslerian features of subacute endocarditis. The pulse was regular at $118 \mathrm{bpm}$, with thickened arterial wall. All peripheral pulses were present and normal. Blood pressure was 142/90 mm Hg from the right arm in cardiac position. Jugular venous pressure was $10 \mathrm{~cm}$ of $\mathrm{H}_{2} \mathrm{O}$ with normal wave forms. A heaving point of maximum apical impulse was felt in the fifth left intercostal space $3 \mathrm{~cm}$ lateral to the mid-clavicular line. There was an $\mathrm{S}_{3}$ gallop of left ventricular origin with 3/6 murmur of mitral regurgitation. There was also a murmur of aortic regurgitation. She had features of right sided pleural effusion. The liver was palpable $2 \mathrm{~cm}$ below the right costal margin with a smooth, sharp and tender edge. A diagnosis of biventricular heart failure was made.

Chest radiograph revealed a cardiothoracic ratio of $53 \%$ with a curvilinear opacity overlying a prominent aortic knuckle and pleural effusion involving the right lower zone. The mediastinum was not dilated. A 12 lead ECG showed a wandering pacemaker with an irregular ventricular rate of 90 cycles per minute, a unifocal PVC of LV origin, QRS axis of +90 degrees and left ventricular hypertrophy by Sokolow's criteria. Transthoracic echocardiogram showed left ventricular enddiastolic diameter of $56 \mathrm{~mm}$ and interventricular septum/posterior wall thickness of $12 / 12 \mathrm{~mm}$. The estimated left ventricular ejection fraction was $40-45 \%$. A SVA involving the non-coronary cusp was visualised in parasternal long axis and short axis views. An apical four chamber view showed the SVA simulating an interatrial septal cyst measuring $38 \mathrm{~mm} \times 40 \mathrm{~mm}$ (Figure 1). The lesion was observed to have an expansile systolic movement, and echocardiography discontinuity was noted on the edge of the aneurysm, resulting in a communication with the left atrium (Figure 2). Doppler flow imaging confirmed a communication between the SVA and LA with the latter serving as the receiving chamber. Mitral annular calcification was observed with a posterior jet of moderate mitral incompetence. Aortic valve had a degenerative morphology with mild aortic regurgitation. There was no aortic stenosis. There was a trivial tricuspid regurgitation. No ventricular or atrial septal defect was detected. Transesophageal echocardiography, cardiac CT/MRI and cardiac catheterization were not available in our centre. Complete blood count revealed a WCC of $5.5 \times 10^{9} / \mathrm{L}$ with a normal differential leucocytes count. The blood film

*Corresponding author: Dr. Mohammed Abdullahi Talle, Department of Medicine, College of Medical Sciences, University of Maiduguri, Nigeria, Tel: +2348035599436; E-mail: abdultalle@yahoo.com

Received March 07, 2018; Accepted March 30, 2018; Published April 06, 2018

Citation: Talle MA, Buba F, Abulfathi A, Baba MM (2018) Ruptured Left Sinus of Valsalva Aneurysm in a Female Nigerian Septuagenarian. J Vasc Med Surg 6: 364 doi: 10.4172/2329-6925.1000364

Copyright: (C) 2018 Talle MA, et al. This is an open-access article distributed under the terms of the Creative Commons Attribution License, which permits unrestricted use, distribution, and reproduction in any medium, provided the original author and source are credited. 
Citation: Talle MA, Buba F, Abulfathi A, Baba MM (2018) Ruptured Left Sinus of Valsalva Aneurysm in a Female Nigerian Septuagenarian. J Vasc Med Surg 6: 364. doi: 10.4172/2329-6925.1000364

Page 2 of 3

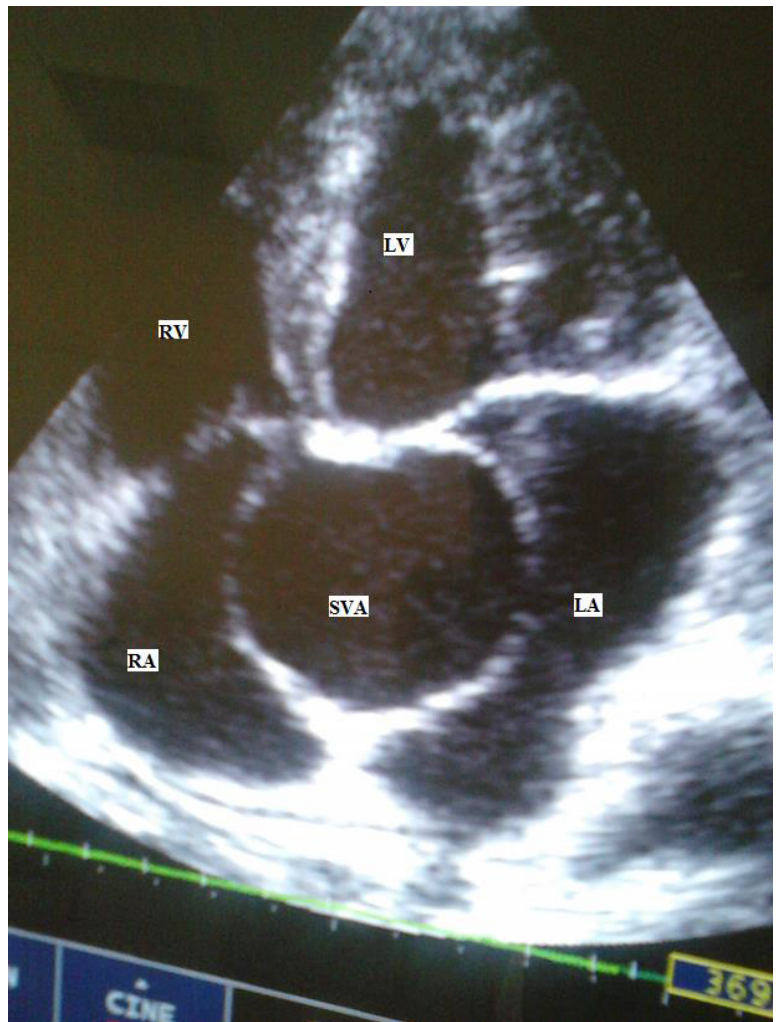

SVA: Sinus of Valsalva Aneurysm; RA: Right Atrium; LA: Left Atrium; RV: Right Ventricle; LV: Left Ventricle

Figure 1: Apical four chamber view of transthoracic echocardiogram depicitng a sinus of valsalva aneurysm enchroaching both right and left atriaum.

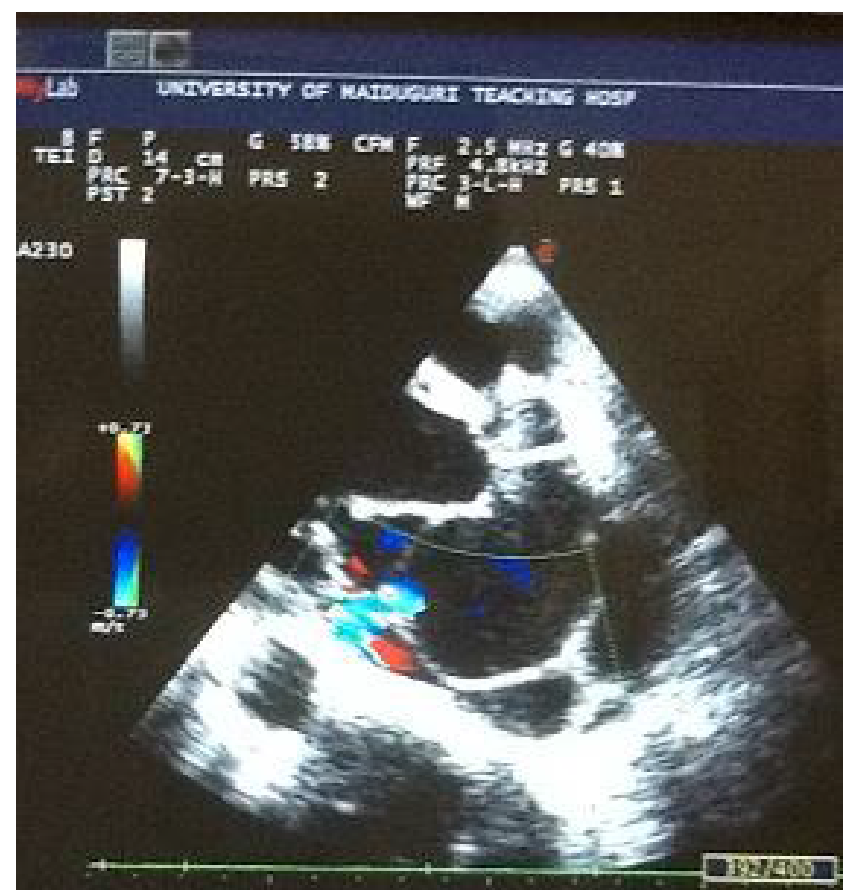

Figures 2: Parasternal long axis view of transthoracic colour flow Doppler echocardiogram showing a communication between the sinus of Valsalva aneurysm and the left atrium. revealed microcytosis and anisocytosis with packed cell volume was $34 \%$. The erythrocyte sedimentation rate was $33 \mathrm{~mm} / \mathrm{h}$.

She was placed on furosemide, lisinopril, spironolactone and made remarkable improvement. Carvedilol was initiated at low dose following the clearance of peripheral edema and pulmonary congestion. She improved to NYHA class II and discharged after two weeks of hospitalization. There was no onsite cardiothoracic surgical service, and referral to a centre with such capability was out rightly rejected by the patient. She remained stable on medical treatment and was followed-up for four months after which the relative reported her sudden unexpected death.

\section{Discussion}

Unruptured SVA is commonly asymptomatic, diagnosed incidentally by echocardiography in adolescents and young adults. However, ruptured SVA presents with murmurs and/or congestive cardiac failure. Few cases have been reported as incidental findings in elderly patients. Our patient presented in her seventies with symptoms of heart failure considered to be due to hypertensive heart disease. Sinus of Valsalva aneurysm was not considered.

Whether our patient had acute heart failure resulting from the ruptured aneurysm or she has had a smouldering background chronic heart failure that did not classically manifest because of age-related limitation of physical activity could not be ascertained. Interestingly, the communication resulting from the rupture did not generate a continuous murmur as expected. This may probably be due to the small nature of the rupture or the fact that it is communicating with the left ventricle since continuous murmur is commonly encountered in ruptures communicating with the right side of the heart.

Common anomalies coexisting with SVA include aortic regurgitation and ventricular septal defects. Although our patient had aortic regurgitation, the aortic valve had morphologic appearance consistent with degenerative valve disease on echocardiography, a common finding in hypertensive septuagenarians.

Unruptured SVA are commonly asymptomatic [1]. Though previously considered an uncommon entity, the advent of echocardiography has led to a more frequent diagnosis of SVA. The presentation in ruptured SVA is quite variable, depending on the size of the rupture, the rapidity with which it develops and the receiving chamber [8]. Two clinical patterns are largely recognised: acute rupture (usually of a large SVA) and the insidiously evolving small perforations $[1,8]$. Abrupt onset of distressing retrosternal chest discomfort, upper abdominal pain, and severe breathlessness with haemodynamic instability characterizes acute rupture of a large SVA. Presentation is subtler in small perforations, remaining asymptomatic for many months, with insidious onset of symptomatic congestive cardiac failure in months to years. Our case highlights the coexistence of ruptured SVA, presumably of the insidious type, with congestive cardiac failure.

Differentiating acquired aneurysm from the congenital forms could be difficult. However, acquired aneurysm from senility involves all the sinuses because of degenerative dilatation, resulting in multiple SVA. In a criterion proposed by Jones and Langely [9], the following findings favour acquired SVA: involvement of an additional SV, extracardiac location, often extending superiorly, and presence of a coexisting cardiac disease (other than congenital heart disease). Some workers alluded that involvement of the left coronary sinus is most consistent with acquired SVA, especially when associated with rupture into the left heart $[5,10]$. 
Citation: Talle MA, Buba F, Abulfathi A, Baba MM (2018) Ruptured Left Sinus of Valsalva Aneurysm in a Female Nigerian Septuagenarian. J Vasc Med Surg 6: 364. doi: 10.4172/2329-6925.1000364

Page 3 of 3

Transthoracic echocardiography is the commonly employed method for the initial evaluation of SVA. Transesophageal echocardiography is highly sensitive for the identification localisation of ruptured SVA as well as assessment of associated abnormalities, especially VSD. 3D transesophageal echocardiography is increasingly being utilized for assessment of SVA, but more importantly, during repair [11]. Other imaging modalities include cardiac catheterization, contrast-enhanced CT scan and cardiac MRI. Our patient only had transthoracic echocardiography.

Surgical intervention remains the treatment of choice for ruptured and symptomatic unruptured SVA [12], with prompt intervention recommended in cases of ruptured SVA to prevent endocarditis or enlargement of the rupture [6]. Although the operative risk associated with repair is generally low, the outcome will greatly depend on patient factors especially in the elderly. Our patient was not subjected to repair because of unavailability of onsite cardiothoracic services, and she declined referral to other centres. She responded to medical treatment of heart failure and was discharged.

The patient was reported to have suffered sudden death after four months of uneventful follow up. Although it was difficult to decipher the cause of her sudden death, especially when no autopsy was done, it may not be closely related to SVA since the rupture in question is deemed insidious. Sudden cardiac death in SVA results from tamponade, myocardial ischemia related to left anterior descending artery ostial occlusion, conduction system defects and arrhythmia $[13,14]$.

This case illustrates the difficulties associated with differentiating between congenital and acquired Sinus of Valsalva aneurysm, an uncommon cardiac problem presenting in the elderly. Although the diagnosis of ruptured SVA aneurysm was made using transthoracic echocardiography, we did not have the benefit of doing additional investigations using transesophageal echocardiography, 3D echocardiography, cardiac catherization, cardiac CT or cardiac MRI. This as well as lack of onsite cardiothoracic services posed major limitations to the management of this case, a prevailing scenario in most of tertiary hospitals of sub-Saharan African countries.

\section{References}

1. Feldman DN, Roman MJ (2006) Aneurysms of the Sinuses of Valsalva Cardiology 106: 73-81.

2. Goldberg N, Krasnow N (1990) Sinus of Valsalva aneurysms. Clin Cardiol 13 831-836.

3. Batiste C, Bansal RC, Razzouk AJ (2004) Echocardiographic features of an unruptured mycotic aneurysm of the right aortic sinus of Valsalva. J Am Soc Echocardiogr 17: 474-477.

4. Medeiros JS, Silva MA, Fontes WF, Santos MA, Pontes SJ, et al. (1989) Syphilitic aneurysm communicating with an aortic sinus of Valsalva. A case report. Arq Bras Cardiol 52: 341-344.

5. Greiss I, Ugolini P, Joyal M, Bouchard D, Mercier LA (2004) Ruptured aneurysm of the left sinus of Valsalva discovered 41 years after a decelerational injury. $J$ Am Soc Echocardiogr 17: 906-909.

6. Takach TJ, Reul GJ, Duncan JM, Cooley DA, Livesay JJ, et al. (1999) Sinus of Valsalva aneurysm or fistula: management and outcome. The Ann Thorac Surg 68: 1573-1577.

7. Smith WA (1914) Aneurysm of the sinus of Valsalva: with report of two cases. JAMA 62: 1878-1880.

8. Golzari M, Riebman JB (2004) The four seasons of ruptured sinus of Valsalva aneurysms: case presentations and review. Heart Surg Forum 7: E577-E583.

9. Jones AM, Langley FA (1949) Aortic sinus aneurysms. Br Heart J 11: 325-349.

10. De MB, Diethrich EB, Liddicoat JE, Kinard SA, Garrett HE (1967) Abnormalities of the sinuses of valsalva. Experience with 35 patients. J Thorac Cardiovasc Surg 54: 312-332.

11. Kumar GA, Parimala PS, Jayaranganath M, Jagadeesh AM (2017) Threedimensional transesophageal echocardiography-guided transcathetar closure of ruptured noncoronary sinus of valsalva aneurysm. Ann Card Anaesth 20 S73-S75.

12. Sawyers JL, Adams JE, Scott HW (1957) Surgical treatment for aneurysms of the aortic sinuses with aorticoatrial fistula: experimental and clinical study. Surgery 41 : $26-42$

13. Munk MD, Gatzoulis MA, King DE, Webb GD (1999) Cardiac tamponade and death from intrapericardial of sinus of Valsalva aneurysm. Eur $\mathrm{J}$ Cardiothorac Surg 15: 100-102.

14. Henry RE, Daisley H, Barton EN (1989) Sudden cardiac death caused by coronary ostial compression by an aneurysm of the sinus of Valsalva. West Indian Med J 38: 250-252. 